\title{
Gestión de residuos de construcción y demolición (RCD) en Bogotá: perspectivas y limitantes
}

\section{Waste management from construction and demolition (RCD) in Bogota: prospects and limitations}

\section{Jesús O. Castaño}

Ingeniero Civil, magíster en Ingeniería Civil. Docente Asistente de la Pontificia Universidad Javeriana. Bogotá, Colombia.

Contacto: jesus.castano@javeriana.edu.co

\section{Rodrigo Misle Rodríguez}

Ingeniero Civil, magíster en Ingeniería Civil. Investigador de la Pontificia Universidad Javeriana. Bogota, Colombia. Contacto: rodrigomisle@gmail.com

\section{Leonardo Andres Lasso}

Ingeniero Civil, magíster en Ingeniería Civil. Investigador de la Pontificia Universidad Javeriana. Bogota, Colombia. Contacto: leonardolasso@gmail.com

\section{Adriana Gómez Cabrera}

Ingeniera Civil, magíster en Ingeniería Civil. Docente Asistente de la Pontificia Universidad Javeriana. Bogotá, Colombia.

Contacto: adrianagomez@javeriana.edu.co

\section{Manuel S. Ocampo}

Ingeniero Civil, doctor en Ingeniería Civil. Docente Asistente de la Pontificia Universidad Javeriana. Bogotá, Colombia.

Contacto:manuel.ocampo@javeriana.edu.co

Fecha de recepción: $\mathbf{3 0}$ de enero de 2013

Clasificación del artículo: revisión de tema

Fecha de aceptación: 15 de abril de 2013

Financiamiento: Vicerrectoría Académica de la Pontificia Universidad Javeriana.

Palabras clave: residuos de construcción y demolición (RCD), gestión de los RCD, agregados reciclados.

Key words: construction and demolition (C\&D) debris, management of C\&D debris, recycled aggregates. 


\section{RESUMEN}

El sector de la construcción en Bogotá ha crecido de forma constante en los últimos años, concentrando entre 20 a $30 \%$ del PIB de la construcción colombiana [3] y con esto se ha venido incrementado la producción de residuos generados. Lo que resulta grave, es la problemática generada por la escases de materia prima cerca al núcleo urbano y el agotamiento de los sitios de vertido autorizados. El reciclaje de residuos de construcción y demolición (RCD) como agregados es una práctica, relativamente difundida en los países desarrollados, para prevenir la contaminación ambiental y disminuir el impacto de la extracción de agregados vírgenes. En Colombia, es un objetivo novedoso que se han venido trazando las entidades públicas encargadas de gestionar la construcción y el medio ambiente. En este artículo se discute el tema de la generación y gestión de los residuos generados por la construcción en Bogotá, además de las políticas publicas y condiciones necesarias para que la actividad del reciclaje de agregados se convierta en una posibilidad viable en nuestro contexto y se generen acciones alrededor de las oportunidades y retos necesarios para alcanzar una gestión total de los RCD.

\section{ABSTRACT}

The construction industry in Bogotá (Colombia) has grown steadily in recent years, concentrated between 20 to $30 \%$ of the GDP of the Colombian construction (2012 Camacol), and this has increased the production of waste. As a consequence, some problems have been presented such as the scarcity of raw materials close to the urban center and the depletion of authorized landfill sites. The waste recycling of construction and demolition (RCD) has been a practical pathway to prevent pollution and reduce the impact of the extraction of Virgin aggregates in developed countries. In our country, waste recycling is a novel target that public companies are still working on, along with the management of construction and environment areas. This article discusses the topic of the generation and management of waste generated by the construction in Bogotá, as well as public policies and conditions necessary for the activity of recycling of aggregates. Also, this article presents the viable possibility of waste recycling in our context and drawn conclusions about the opportunities and challenges required to achieve a total management of the RCD.

\section{INTRODUCCIÓN}

Los residuos de construcción y demolición (RCD) son materiales de desecho, generados en las actividades de construcción, demolición y reforma, de edificaciones, obra civil y espacio público. Estos son considerados inertes -no peligrosos- y poseen alta susceptibilidad de ser aprovechados mediante transformación y reincorporación como materia prima de agregados en la fabricación de nuevos productos. Actualmente, se producen en Bogotá, cerca de 15 millones de ton/año de RCD [6], algo como $2000 \mathrm{~kg}$.hab/año, una cifra alar-

mante, situándonos incluso por encima de grandes urbes en el mundo y convirtiéndose en uno de los principales problemas que impactan el ambiente de la ciudad. De acuerdo a lo anterior, el Distrito ha encaminado esfuerzos y recursos con el ánimo de mejorar la gestión de RCD, incorporando normas y regulaciones, aunque con importantes carencias y limitaciones, las cuales, si no se resuelven en el corto plazo, el proceso encaminado a mejorar la sostenibilidad del sector se interrumpe y las medidas tomadas hasta el momento se vuelven ineficaces. 
Este trabajo pretende mostrar lo concerniente a un adecuado proceso de manejo y transformación de los RCD, poniendo de manifiesto los problemas que se pueden generar a fin de evitarlos, de forma que todos los agentes implicados conozcan el importante papel que desempeñan en la correcta gestión.

\section{ANTECEDENTES}

El sector de la construcción ha crecido de forma constante en la última década, y con él, la producción de residuos de construcción y demolición (RCD). Para dar una idea de la importancia de la generación de estos residuos, cabe mencionar que la estimación aportada por la Secretaria Distrital de Planeación y Ambiente de Bogotá, de 2 toneladas per cápita, representa una magnitud de producción alarmante al ser comparada con el promedio europeo antes de la crisis del 2010, solo países como Dinamarca, Finlandia, Alemania, Irlanda y Luxemburgo, presentaron cantidades superiores a 2 ton/año per cápita. Más desalentador resulta el panorama si revisamos las cifras de reutilización de estos mismos países, los cuales superan el 50\% de reutilización de RCD [2].

De este gran volumen de RCD que se producen en Bogotá, los mayores productores son el IDU y las construcciones privadas, como se observa en el figura 1, y en muchos casos, van a parar a sitios no autorizados, alterando el paisaje, contaminando suelos y acuíferos.

Esto también representa una pérdida de recursos potenciales, pues el hecho de que se desechen como residuos ciertos elementos provenientes de las obras, que poseen aún capacidad de ser valorizados, además de que obliga a consumir recursos naturales, acentua más el efecto negativo de la actividad constructora en Bogotá.

Hace algunos años, no se habría pensado en reutilizar escombros de construcción como agregados

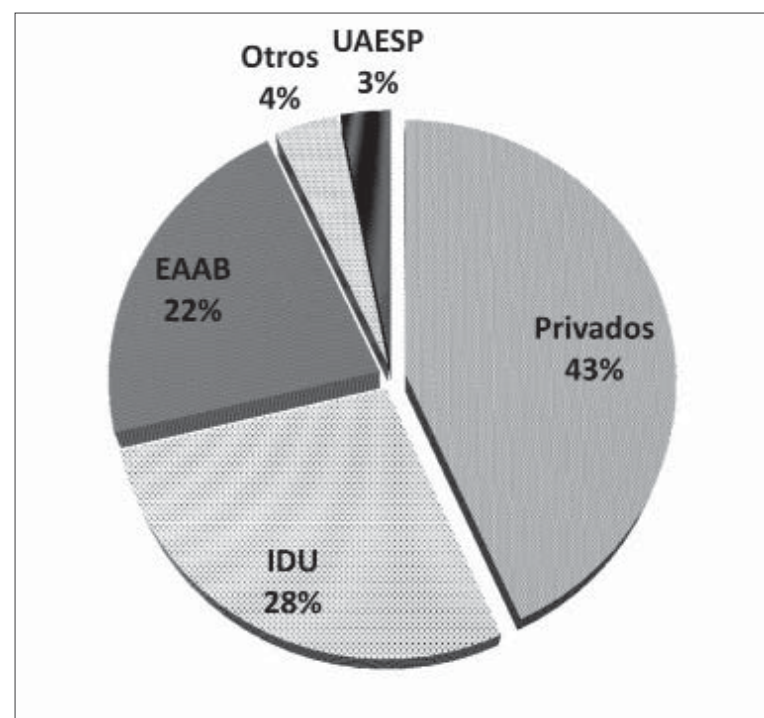

Figura 1. Generadores de RCD en Bogotá

Fuente: tomado de [6].

para nuevos materiales, dada la gran oferta de materias primas en la ciudad, no obstante, alrededor del mundo, múltiples investigaciones han mostrado que estos, pueden sustituir de forma satisfactoria los agregados naturales, como una manera de transformar la industria de la construcción en una actividad sostenible.

De hecho, se ha propiciado la aparición de normas legales que regulen la producción y gestión de RCD en Colombia en los últimos años, incluso desde la fase de redacción de proyecto, con lo que se pretende garantizar la mejora la situación ambiental en la ciudad [6]. La finalidad específica de esas normas implica: la reducción de la producción de residuos, la reutilización de aquellos residuos o elementos que así lo permitan, el reciclado de los residuos que no puedan reutilizarse, la valorización energética de los residuos que no puedan reciclarse y el depósito adecuado en vertedero de todo lo que no pueda valorizarse.

La aparición de estas normas podría frenar el deterioro del medio en el que nos vemos inmersos, pero esto implica un cambio drástico en la mentalidad de todos los agentes implicados, siendo im- 

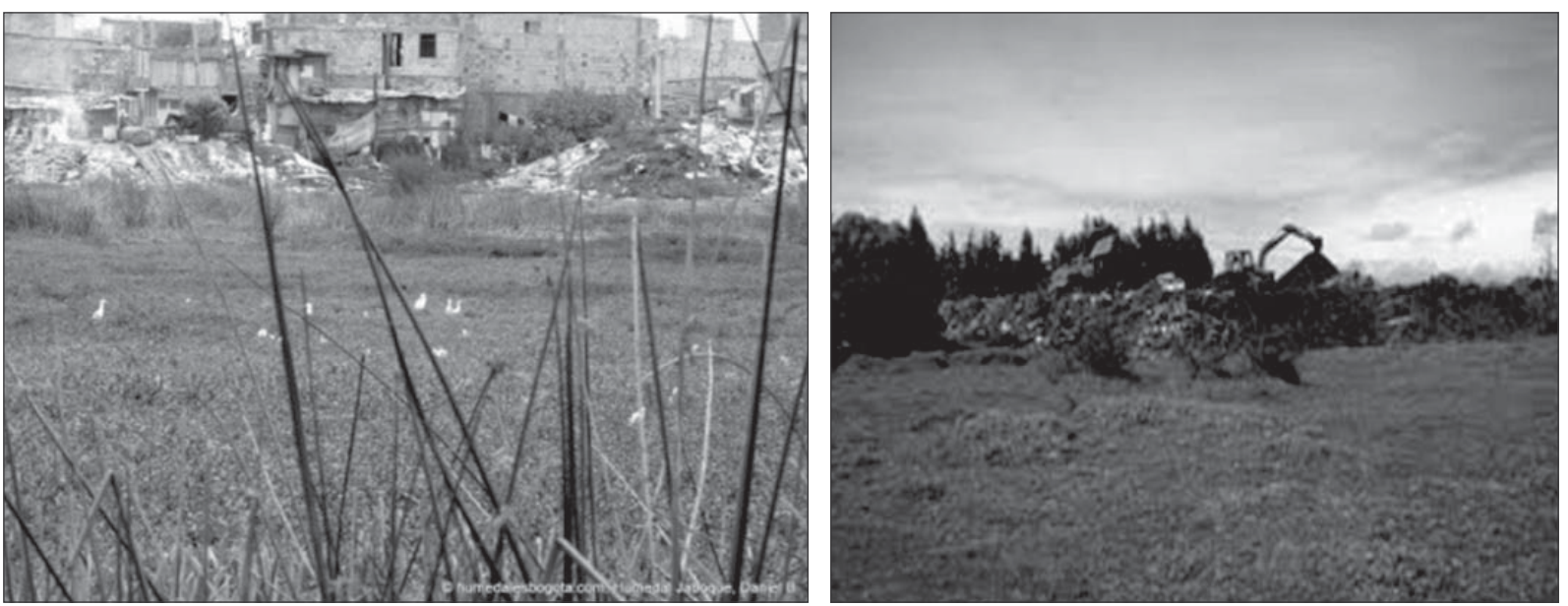

Figura 2. Contaminación de humedales

Fuente: disponible en http://humedalesbogota.com

prescindible su compromiso activo, aportando así una pequeña contribución que, sumada a otras, como la certificación PRECO [6], para edificaciones, permita acercarse a la sostenibilidad global de la actividad constructora.

Hasta ahora, la opción más sencilla consiste en deshacerse de los residuos depositándolos en vertederos legales, en el mejor de los casos, sin embargo, esta opción no es sostenible, pues es inminente el agotamiento de los sitios de disposición cercanos al casco urbano, de hecho, en los últimos años la CAR (Corporación Autónoma Regional de Cundinamarca) ha detectado la presencia de 94 sitios de disposición ilegal de escombros en Bogotá y alrededores, que en algunos casos han ido destruyendo los humedales.

Se pretende que, en el futuro, tampoco sea ventajosa la disposición de residuos con potencial de reutilización desde un punto de vista económico, desincentivando la mala gestión de residuos a través de la aplicación de tasas cada vez mayores para los residuos segregados, como ya es política en otros países. Lo adecuado es separar los diferentes residuos en obra y entregar los RCD a un gestor autorizado para su total valorización.

\section{ACTUALIDAD DEL RECICLAJE DE RCD EN BOGOTÁ}

En el Distrito, al igual que en el resto del país, los RCD tiene como sistema de gestión el vertido -controlado en contadas ocasiones, pero sobre todo incontrolado-, los sitios autorizados están totalmente diseminados, siendo escasa cualquier otra alternativa de valorización, reciclaje o reutilización, poco más del $5 \%$ a $10 \%$ de este tipo de residuos son sometidos a procesos de reciclaje y reutilización, en un par de empresas que a la vez que expiden certificado de disposición legal de escombros, comercializan productos granulares que cumplen con la normativa colombiana para uso en construcción.

Otro caso son los llamados "Molineros" (figura 3) ubicados en la zona sur de la ciudad, que tienen un programa de recuperación de materiales de construcción artesanal, mediante la producción de arenas producto de la trituración de RCD; sin embargo, la calidad de sus productos en su mayoría no cumplen con las normativas para utilización en construcción, no poseen programas de manejo ambiental y las condiciones laborales y de salud ocupacional son deficientes [8]. 

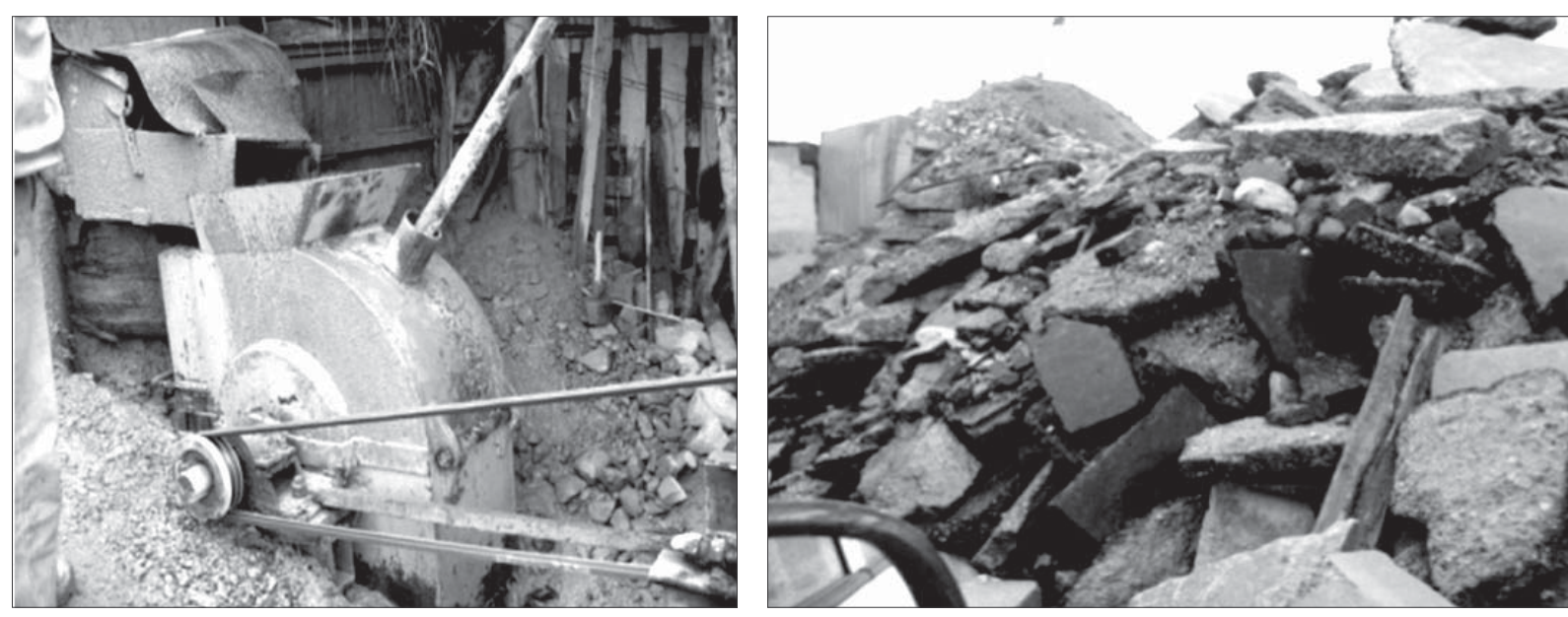

Figura 3. Reciclaje por "Molineros" en el sur de Bogotá

Fuente: tomado de [8]

En el 2011, la Secretaria de Ambiente Distrital expide la resolución 2397, la cual obliga a que las constructoras bogotanas aprovechen los residuos de sus obras, evitando así que lleguen a humedales, ríos, quebradas o al espacio público. Esta medida contempla gradualidad, puesto que el porcentaje de RCD a reutilizar inicialmente exigido es del $5 \%$ y cada año los porcentajes serán incrementados en cinco unidades hasta alcanzar un $25 \%$. No obstante a los actores que incumplan se les sancionaría con una contravención de carácter ambiental que puede llegar a $5000 \mathrm{smmlv} /$ diarios (\$2800 mill de 2012), además del cierre temporal o definitivo del establecimiento. Y tal vez lo más llamativo de dicha resolución es que aporta a la posibilidad de negocio, al promover el uso de los centros de aprovechamiento, donde se tratarán los residuos de construcción y demolición para crear productos comerciales.

\section{FUNCIONAMIENTO DE UNA PLANTA DE TRANSFORMACIÓN DE RCD}

El esquema de funcionamiento básico de una planta de tratamiento es el siguiente: al ingresar los camiones volqueta, procedentes de las diferentes obras de la ciudad, se procede a una inspección visual de la mezcla, pesaje y determinación del tratamiento posterior del residuo. En esta zona es donde se fijará el costo de la gestión, dado que el material separado desde obra tendrá algún incentivo.

Una vez el residuo es aceptado, pasa a la zona de descarga para la separación por medios manuales o mecánicos de los distintos componentes, para posteriormente introducirla a la línea de trituración y separación, compuesta por machacadora de mandíbulas, triaje manual, sistemas de cribado y zona de acopio final por fracciones granulométricas. Estos agregados pueden ser empleados en constitución de bases, subbases y mezclas hidráulicas. La figura 4 muestra el esquema de la planta de procesamiento de RCD.

Este tipo de plantas de transformación debe ser capaz de separar, y en su caso tratar, otros residuos como: madera, hierro, envases metálicos y plásticos, cartón, papel, mobiliario doméstico, vidrio, pinturas y aceites, para ser entregados a gestores autorizados.

Finalmente, el residuo que no se puede valorizar o transformar, se debe llevar a un sitio de disposi- 


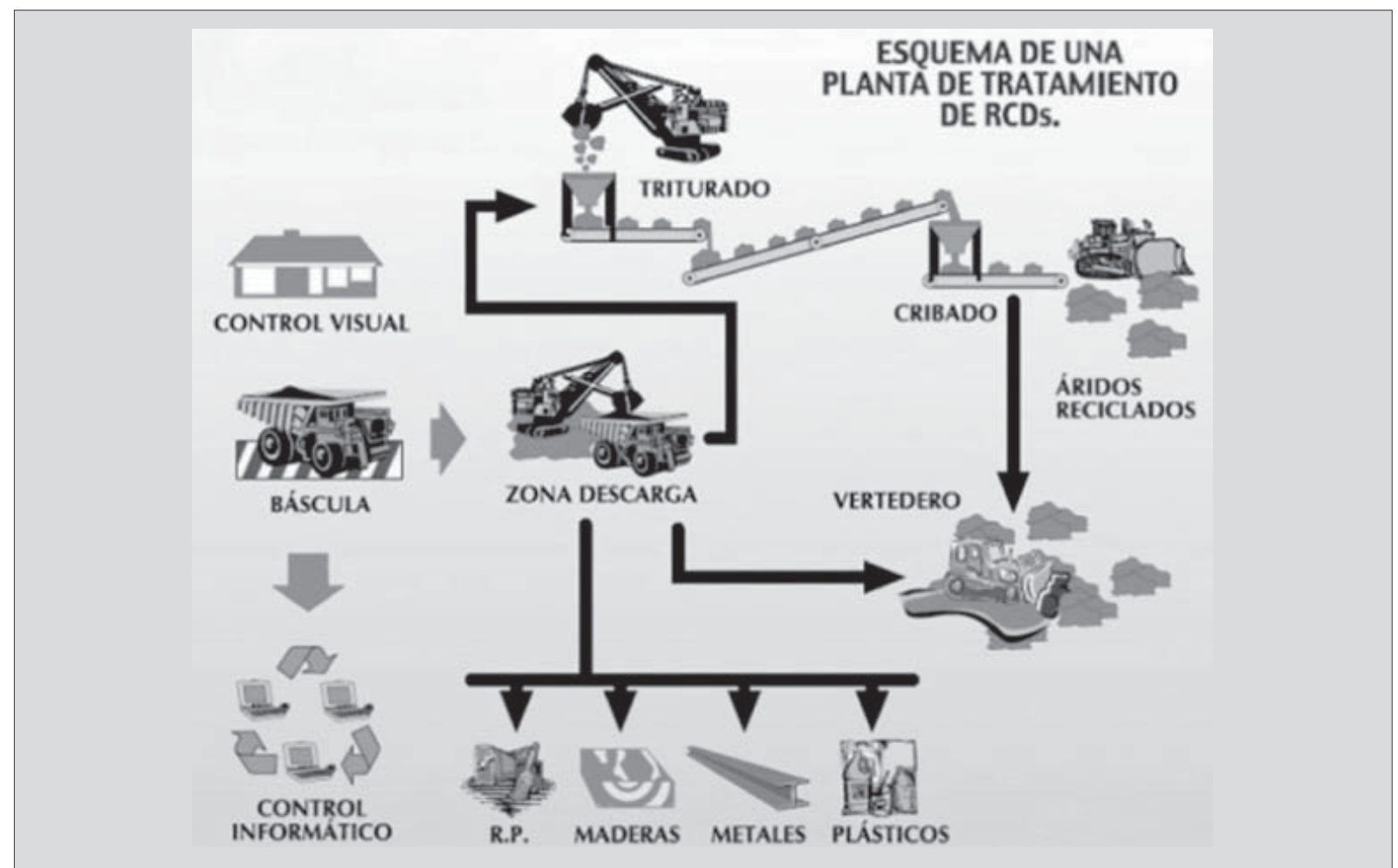

Figura 4. Esquema de planta de procesamiento de RCD

Fuente: tomado de [8]

ción final, preferiblemente en zonas de recomposición paisajística, tal como se viene adelantando en el lote de La Fiscala (figura 5).

Localizada en el kilómetro 6 vía Usme y con una área de 20 ha, corresponde a una mina de agregados que finalizó su etapa productiva en el 2004 y ahora se convierte en Reserva ecológica privada, posterior a una reconformación morfológica y ambiental de la antigua área de operaciones y a un proyecto de gestión social [5].

Si se desea hacer un plan de gestión de RCD (figura 6) completo, se deberá recurrir a un sistema de recolección de los escombros en las mismas obras, con el fin de que esta operación sea más ordenada y para ello se puede recurrir a dos tipos de sistemas principalmente: los camiones tipo
Roll-off y los camiones tipo bañera; sin embargo, el tipo Roll-off por su capacidad llega a ser más eficiente.

\section{ESTUDIO ECONÓMICO DE LA GESTIÓN DE RCD EN EL DISTRITO}

La UAESP en el 2009, presenta los resultados de un estudio de prefactibilidad para la gestión de escombros en el Distrito, precisando que, del volumen total de escombros para el 2013 (UAESP); $14000 \mathrm{de}^{3}$ /dia, solo un $30 \%$ sería susceptible a reciclar, pues el $70 \%$ restante se compone de tierras. En este análisis se decide optar por un sistema de trituración con capacidad de 150 Ton/hora, 75 chasis roll-off, 350 contenedores y 50 remolques, todo esto le cos- 


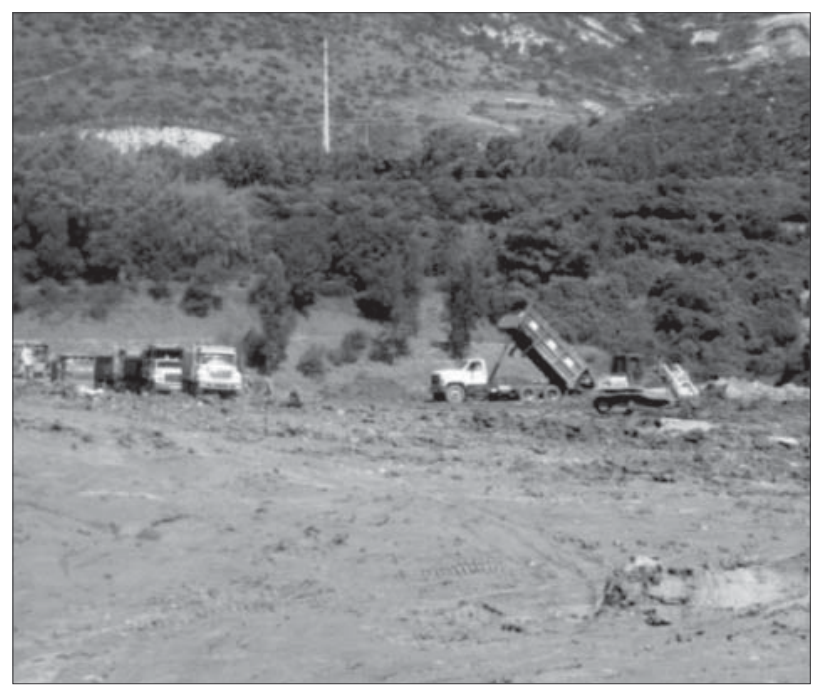

Figura 5. Adecuación paisajística La Fiscala

Fuente: elaboración propia

taría al Distrito la suma de $\$ 33675$ millones, sin embargo, aunque la solución presentada es integral, queda pendiente un estudio detallado de factibilidad.

Posteriormente, el estudio realizado por Misle y Lasso [4] en la Pontificia Universidad Javeriana, revela un análisis detallado muy importante el cual permitirá que no solo el Distrito pueda plantearse la opción de sacar adelante el proyecto sino que presenta un amplio estudio económi-

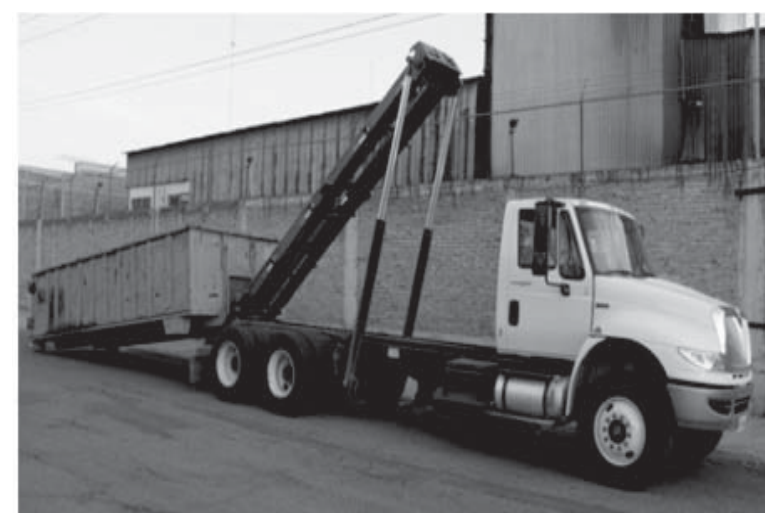

Figura 6. Contenedores para recolección de RCD Fuente: elaboración propia

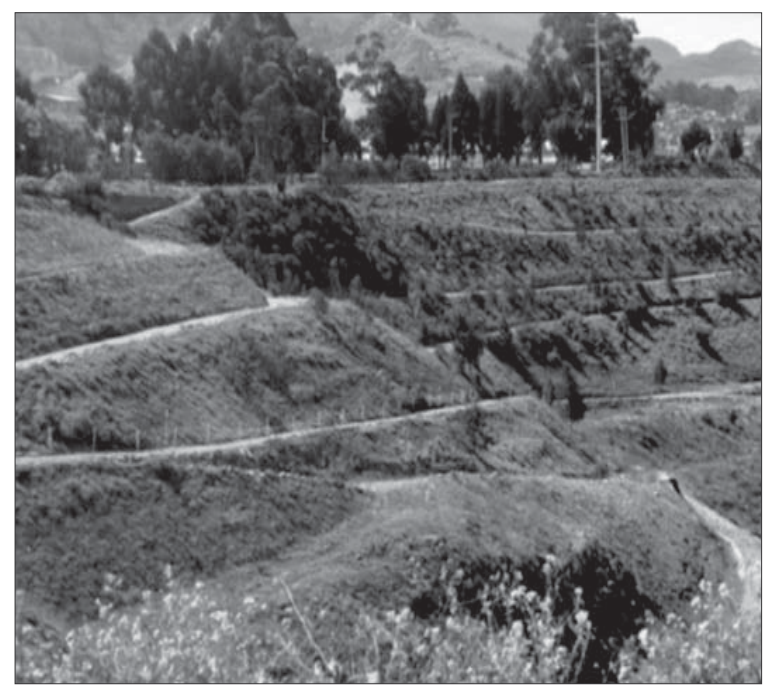

co con miras a que un privado invierta en un negocio rentable tal como promueve la resolución 2397 de 2011 de la SDA.

En resumen, en el estudio realizado por Misle y Lasso, concluyen que el volumen de escombros para el 2013 (UAESP); 15 millones de $\mathrm{m}^{3}$ /año, representaría unos $50000 \mathrm{~m}^{3} /$ dia, pero solo un volumen susceptible a reciclar del $15 \%$ que es el correspondiente a prefabricados y demolición de morteros y concretos, esto con miras a producir un agregado de óptima calidad, a su vez plantean una empresa de gestión que se encargue del procesamiento de un $10 \%$ del RCD, algo así como $225000 \mathrm{~m}^{3}$ / año, para poder llevar a cabo esta tarea con un equipo de 150 Ton/hora similar al propuesto por Coambiente en el 2009.

En el estudio mencionado, se proponen tres modelos de empresa, siendo el primer modelo el modelo aplicable a una empresa del Distrito, la cual con una inversión privada de aproximadamente 11000 millones (100\% de inversión) en 7 años se le devuelve el capital al inversor con una tasa social de descuento DNP del $12 \%$ 


\section{revisión |}

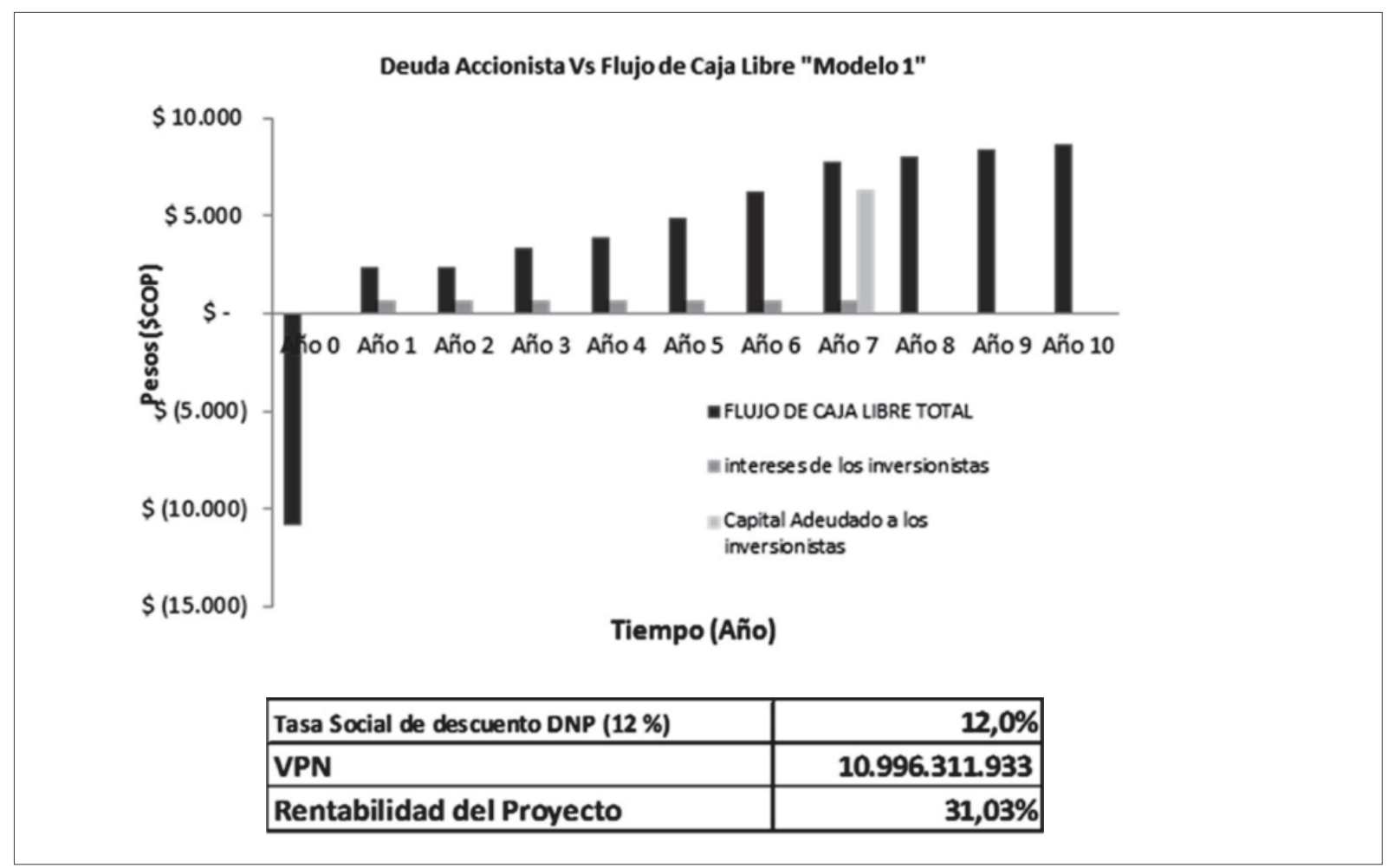

Figura 7. Resultados de Modelo Económico empresa mixta

Fuente: tomado de [4].

y la empresa en 10 años produce una rentabilidad mayor al $30 \%$, lo cual resulta en un negocio muy atractivo.

\section{CONCLUSIONES}

La conclusión principal en este estudio es la necesidad apremiante de una solución al tema de los RCD en el Distrito, y convenientemente debe ser la administración la que proporcione las herramientas que permitan concluir satisfactoriamente el proceso, creando plantas de transformación de primer nivel y zonas de almacenamiento o vertederos de residuos no peligrosos situados en zonas aisladas.
De acuerdo con estudios consultados, la alternativa de sustituir agregados vírgenes por reciclados resulta viable técnicamente, dado que las propiedades que exiben estos últimos cumplen con la normativa actual de agregados para construcción, en cuanto a bases, subbases, lechadas y mezclas de concreto hidráulico y asfaltico.

En el estudio presentado por Misle y Lasso, las cifras económicas resultan muy atractivas para hacer de los modelos propuestos unas empresas rentables, sin embargo, este entramado de gestores de residuos debe estar presente en número adecuado para que la aplicación del proceso sea viable. 


\section{REFERENCIAS}

[1]. Alcaldia de Bogotá, Avance de Formulación - Política Pública de Edificaciones Sostenibles, Secretarias Distritales de Planeación y Ambiente, Bogotá, 2011

[2]. I. Bio Service, "Service Contract on Management of Construction and demolition Waste", European Commission (DG ENV), Paris, 2011.

[3]. Camacol, Construcción en cifras, Bogota, 2012.

[4]. R. Misle y L. Lasso, "Evaluacion Tecnica, Economica e Institucional de la Gestion de Residuos de Construccion y Demolicion en la Ciudad de Bogota", Pontificia Universidad Javeriana, Bogotá, 2012.

[5]. C. S. Ramirez, "Diagnostico sobre estado y manejo de escombros en el distrito Capital
Bogota; Estudio de caso escombrera reserva ecologica privada La Fiscala", Facultad de estudios Ambientales, Universidad Javeriana. Bogotá, 2009.

[6]. SDA, Resolucion 2397. Por la cual se regula técnicamente el tratamiento y/o aprovechamiento de escombros en el Distrito Capital, Secretaria Distrital de Ambiente, Bogotá, 2011.

[7]. SDA, Resolucion 5926. Por la cual se crea $y$ regula el programa de reconocimiento ambiental a Edificaciones Ecoeficientes PRECO-, Secretaria Dstrital de Ambiente, Bogotá, 2011.

[8]. UAESP, Informe Censo de Molineros, Bogota, 2010. 